\title{
Analysis of Incident DKA in the Indiana New Onset T1D Patient Population
}

Brianna Beer ${ }^{1}$, Kelly Moors ${ }^{2,3}$, Carmella Evans-Molina ${ }^{2,3}$, Emily K. Sims ${ }^{2,3}$

${ }^{1}$ Marian University School of Osteopathic Medicine, ${ }^{2}$ Indiana University School of Medicine, Department of Pediatrics and the Herman B Wells Center and the ${ }^{3} \mathrm{UU}$ Center for Diabetes and Metabolic Diseases

Background/Objective: Diabetic ketoacidosis (DKA) is a life-threatening complication of type 1 diabetes (T1D) resulting from ketone body production and metabolic acidosis occurring due to insulin deficiency. We sought to define the occurrence of DKA amongst pediatric patients presenting with new-onset T1D in Indiana and to determine whether patterns of DKA were affected by the COVID-19 pandemic.

Methods: This was a retrospective chart review for patients $<18$ years admitted to Riley Children's Hospital with a clinical diagnosis of new onset T1D who had available chemistry values. Patients diagnosed from March 23- June 30, 2020 and over the same period in 2019 were included. DKA was classified as mild (bicarbonate $10-15 \mathrm{mmol} / \mathrm{L}$ ) or severe (bicarbonate $<10 \mathrm{mmol} / \mathrm{L})$.

Results: Ninety-four patients met inclusion criteria. The total number of incident T1D cases in 2019 and 2020 were similar (48 vs. 46, respectively). Similarly, there was no significant difference in rates of DKA (21 in 2019 vs. 25 in 2020; p>0.05). Of the 94 patients, 49\% met criteria for DKA; $79 \%$ of cases were classified as severe and $21 \%$ as mild. More males were diagnosed with DKA in both 2019 and 2020 (61\% of DKA cases). Non-Hispanic whites comprised $75 \%$ of all new onset T1D patients and no differences in race or ethnicity were present amongst those presenting in DKA.

Conclusion: DKA was present in nearly half of all new onset pediatric T1D cases in Indiana in 2019 and 2020. There was no observed impact of the COVID-19 pandemic on T1D or DKA.

Impact and Implications: DKA is common amongst pediatric patients with new onset T1D in Indiana. Prompt recognition of symptoms is needed to prevent this life-threatening complication of T1D. 